Revista de Psicología Vol. 36 (2), 2018 (ISSN 0254-9247)

\title{
Bullying homofóbico en México a nivel de secundaria: el contexto de Nuevo León
}

\author{
Luis M. Rodríguez Otero ${ }^{1}$ \\ Universidade de Santiago de Compostela, España
}

En el entorno escolar la orientación sexual pude convertirse en un elemento que favorece la existencia de bullying entre el estudiantado. Se propone este estudio con el objetivo de identificar su existencia y sus características en las escuelas secundarias de Monterrey-México, por cuanto no se reportan estudios sobre el bullying homofóbico en Nuevo León. A través de una metodología mixta y un muestreo intencionado se analizó el bullying homofóbico mediante un instrumento formado con preguntas abiertas y cerradas a 330 estudiantes. Los resultados revelan que el $44,54 \%$ reconoce haber presenciado bullying en su escuela $(31,29 \%$ referentes a la orientación sexual). Asimismo, se identificaron siete víctimas de bullying homofóbico. Es por ello que se considera necesario proponer medidas legales, sociocomuntarias y educativas preventivas.

Palabras clave: bullying, violencia, secundaria, México, homofobia.

\section{Homophobic bullying in Mexican secondary level: Nuevo Leon context}

Sexual orientation can become an element that favors the existence of bullying among students in the school environment. As there is no research in Nuevo Leon on homophobic bullying, this study aims to identify its existence and characteristics in the high schools of Monterrey, México. Through a mixed methodology and intentional sampling, 330 students were surveyed using an instrument with both open and close-ended questions. The results reveal that $44.54 \%$ of participants have witnessed bullying in their school $(31.29 \%$ related to sexual orientation). In addition, we identified seven victims of homophobic bullying. It is necessary to propose legal, socioecological and preventive educational measures.

Keywords: bullying, violence, high school, Mexico, homophobia.

\section{Bullying homofóbico no México no nível secundário: o contexto de Nuevo León}

No ambiente escolar, a orientaçáo sexual pode se tornar um elemento que favorece a existência de bullying entre os estudantes. Não existindo pesquisas sobre o bullying homofóbico

1 Trabajador social. Doctor en Menores en Situación de Desprotección y Conflicto Social por la Universidad de Vigo (España). Profesor-investigador/a de la EUTS- Universidade de Santiago de Compostela. España. Dirección postal: Calle Ramón Cabanillas n ${ }^{\circ} 16-4^{\circ}$ CP 32004 - Ourense, Galicia, España. Contacto: luismaotero@yahoo.es 
em Nuevo León se propóe este estudo, com o objetivo de identificar sua existência e características em escolas secundárias de Monterrey, México. Por meio de uma metodologia mista e com uma amostragem intencional, foi aplicado um instrumento de coleta de dados, formado por perguntas abertas e fechadas, para 330 alunos. Os resultados mostram que $44,54 \%$ reconhecem ter testemunhado bullying na escola $(31,29 \%$ relativa a orientação sexual). Os participantes também identificaram sete vítimas de bullying homofóbico. É por isso que considera-se necessário propor medidas preventivas aos níveis legal, sócio-comunitario e educacional.

Palavras-chave: bullying, violência, secundário, México, homofobia.

\section{Bullying homophobe au Mexique au niveau secondaire: le contexte de Nuevo León}

Dans le milieu scolaire, l'orientation sexuelle peut devenir un élément favorisant bullying dans le corps étudiant. Il n'y a pas de recherche sur bullying homophobe au Nuevo Leon, cette étude est proposée dans le but d'identifier son existence et ses caractéristiques dans les écoles secondaires de Monterrey, au Mexique. L'utilisation d'une méthodologie mixte et d'un échantillonnage intentionnel a été analysée à travers un instrument formé de questions ouvertes et fermées à 330 étudiants. Les résultats révèlent que 44,54\% admettent avoir été témoin d'intimidation dans leur école (31,29\% se référant à l'orientation sexuelle). De même, 7 victimes d'intimidation homophobe ont été identifiées. C'est pourquoi il est jugé nécessaire de proposer des mesures préventives légales, sociocommunautaires et éducatives. Mots-clés: bullying, violence, secondaire, Mexique, homophobie. 
Bullying homofóbico en México a nivel de secundaria: el contexto de Nuevo León / Rodríguez O.

El bullying es el abuso, maltrato o violencia física, verbal, gesticular y/o tecnológica entre iguales que tiene lugar en el entorno escolar, por uno o varios agresores/as hacia otro compańero/a, que se producen de forma repetida e intencionada, y que atentan contra la integridad física, material o emocional de las víctimas (Vega, 2013; Olweus, 1998). En referencia a las víctimas, diversos autores ponen de manifiesto que estas suelen presentar dificultades para hacer frente, por sus propios medios, a dichas situaciones de violencia o abuso. Asimismo también destacan los importantes efectos negativos que dichas situaciones producen en las víctimas. Destacando principalmente: una baja autoestima, cuadros de ansiedad y/o depresivos, dificultades de aprendizaje e integración escolar, riesgo de suicidio, fracaso y/o deserción escolar (Serrate, 2007; Cobo \& Tello, 2010; Moratto, Cárdenas \& Berbesí, 2012).

En la existencia de este tipo de actos o actitudes, tal y como señalan autores como Agustín (2009), existen ciertos factores que resultan clave, especialmente: el clima de convivencia en el centro, las características sociodemográficas y contextuales del alumnado, la orientación pedagógica del centro educativo y la existencia de vías y/o protocolos de preveción e intervención ante este tipo de situaciones. Por otro lado, cabe destacar que autores como Gualdi et al. (2008) señalan que las principales causas por las que los agresores hacen uso del bullying derivan de la presencia de determinados rasgos (físicos, del lenguaje y/o comportamentales), así como de la pertenencia a determinadas comunidades minoritarias y/o grupos socialmente estigmatizados, entre los que se encuentran las personas lesbianas, gays, transexuales, bisexuales e intersexuales (LGTBI).

Producto de la homofobia, en el entorno escolar se ejerce violencia psicológica, verbal y física entre iguales hacia personas LGTBI e incluso heterosexuales (que no comparten el perfil heteronormativo). Esto se manifiesta como medio de condena a comportamientos o actitudes que difieren del modelo heterocentrista (Gualdi et al., 2008; Samaniego \& 
Bermúdez, 2015; García, 2015). Este tipo de situaciones se denomina bullying homofóbico, el cual refiere, tal y como señala Agustín (2009) a:

Cualquier tipo de agresión verbal, física o social entre estudiantes que: bien está destinada a atacar a determinadas personas por su orientación sexual real o percibida o la de algún familiar o amigo de la víctima. O bien se produce con otra motivación (por ejemplo: aumentar el estatus en el grupo), pero se ampara en la supuesta orientación sexual de la víctima por considerarla especialmente vulnerable. (p. 83).

También añade que se caracteriza por "la intención de hacer daño (físico o psicológico), la reiteración de las conductas y el desequilibrio de poder que hace a la víctima impotente para salir de esa situación por sí sola” (Agustín, 2009, p. 83).

Por otro lado, cabe señalar que el bullying homofóbico se caracteriza por ciertos rasgos distintivos con el resto de tipologías de acoso escolar entre iguales, destacando especialmente: (i) la existencia de lenguaje homofóbico con gran calado, (ii) la exclusión par parte de ciertos colectivos/grupos a las personas LGTBI, (iii) la invisibilidad de la diversidad sexual en las aulas y/o la falta de una orientación definida en la adolescencia por parte de algunos menores, (iv) la existencia de mitos y estereotipos socialmente aceptados respecto a las personas LGTBI, (v) el hecho de que el entorno social y/o familiar desconozca la orientación sexual de la víctima, (vi) el posible calado de la LGTBI-fobia en el profesorado, equipo directivo del centro, la familia del menor y/o el propio alumnado, (vii) la posible existencia de contenidos LGTBI-fóbicos en los currículums académicos y/o explicaciones del profesorado, (viii) la interiorización de la LGTBI-fobia en las víctimas y el temor a denunciar los hechos como vía de evidencia de la propia orientación sexual oculta, (ix) la transferencia del estigma a los compañeros que defienden a quienes son objeto de bullying, $(\mathrm{x})$ la inexistencia de referentes de ayuda entre la población LGTBI y (xi) el temor de las víctimas a ser rechazadas por el profesorado (Gualdi et al., 2008; Samaniego \& Bermúdez, 2015; Birkett, Espelage \& Koenig, 2009; Mooij, 2015; Kolbert 
et al., 2015; García, 2015; Martxueta \& Etxeberría, 2014; Poteat \& Espelage, 2005).

Este tipo de violencia se caracteriza por presentar una prevalencia relativamente alta que depende en gran medida del contexto social, la aceptación que existe hacia la diversidad sexual y las políticas existentes de lucha contra la LGTBI-fobia (FELGTBI, 2007; COGAM, 2013; Gualdi et al., 2008). Así, se identifican estudios que indican que un $20 \%$ del estudiantado LGB — lesbianas, gays y bisexuales — sufren bullying (Rivers \& Soutter, 1996; De Souza, Pereira \& Ferro, 2015) y otros señalan que la prevalencia se sitúa entre un 30 y un 60\% (Martxueta \& Etxeberría, 2014; Antonio, Pinto, Pereira, Farcos \& Moleiro, 2012). Sin embargo, también se han publicado estudios que evidencian niveles superiores al 80\% (Samaniego \& Bermúdez, 2015; D’Augelli, Pilkington \& Hershberger, 2002). Es por ello que destaca el estudio realizado por Gualdi et al. (2008) en secundarias de España, Italia, Austria y Polonia, a través del cual identifican que, dependiendo del país, entre un 50 y un $75 \%$ del estudiantado reconoce haber presenciado bullying homofóbico.

Por otra parte, cabe señalar que, al igual que indican los estudios referentes al bullying a nivel general, este tipo de hostigamiento/ violencia se caracteriza por derivar en distintas consecuencias en las víctimas tales como depresión, ansiedad, peor autoconcepto o baja autoestima (Burton, Marshal \& Chisolm, 2014; Pachankis, Goldfried \& Ramrathan, 2008; Martxueta \& Etxeberría, 2014; Antonio, Pinto, Pereira, Farcos \& Moleiro, 2012), así como empeoramiento del rendimiento académico, fracaso y/o deserción escolar (Poteat, Mereish, Digiovanni \& Koenig, 2011; Burton, Marshal \& Chisolm, 2014; Bastida, 2010) y también mayor riesgo de suicidio (Teacher, 2003 en Gualdi et al., 2008; Gualdi et al., 2008; Poteat et al. 2011; Baiocco, Loverno, Lonigro, Baumgartner \& Laghi, 2015; Rivers, 2001; Bastida, 2010).

Otro aspecto importante a la hora de abordar el bullying homofóbico es el referente al perfil tanto de las víctimas como de los agresores. Estudios, como los realizados por Gualdi et al. (2008), Martxueta y 
Etxeberría (2014), De Souza, Pereira y Ferro (2015) o Antonio, Pinto, Pereira, Farcos y Moleiro (2012), señalan que a pesar de que se produce tanto por hombres como por mujeres, este tipo de actitudes poseen un mayor calado en el estudiantado masculino. También se observa que son los gays en mayor medida objeto de ataques y/o hostigamiento por parte de sus compañeros/as. Asimismo, se observa que la violencia verbal y la psicológica son las vías más frecuentes a través de las cuales se materializa este tipo de bullying (Antonio, Pinto, Pereira, Farcos \& Moleiro, 2012; De Souza, Pereira \& Ferro, 2015; Gualdi et al., 2008; D’Augelli, Pilkington \& Hershberger, 2002).

\section{El contexto mexicano}

Tomando en consideración el contexto mexicano, Bastida (2012) señala que el bullying homofóbico es una realidad en las escuelas mexicanas. Así realiza un análisis de distintas publicaciones en periódicos que evidencian cinco casos concretos en la Ciudad de México, Aguascalientes y Tabasco. Por otro lado, el Informe Nacional sobre Violencia de Género en la Educación Básica en México (México, 2009) indica que la Secretaría de Educación Pública (SEP) evidenció que el 22\% del estudiantado de Educación Básica se identifica como agresores de bullying homofóbico. Asimismo, Granados-Cosme y Delgado-Sánchez (2008), mediante entrevistas a una muestra de 19 homosexuales de una universidad de la Ciudad de México, observaron manifestaciones de violencia verbal: burlas, acoso y agresiones físicas, las cuales se producían principalmente por la forma de hablar de las víctimas y/o por ser identificadas como afeminados. También destaca que las víctimas reconocen la existencia de consecuencias derivadas de esta situación tales como sentimientos de culpa, autorrechazo, tristeza, aislamiento, depresión e ideas o intentos de suicidio. Del mismo modo, Lozano (2014), mediante un estudio cualitativo realizado mediante entrevistas a profundidad a 15 hombres gay de la Ciudad de México, observó que toda la muestra manifestaba haber sufrido bullying mediante violencia verbal y asilamiento en su etapa formativa (especialmente por 
relacionarse con chicas) y que este tuvo efectos negativos en su vida. También Quintanilla, Sánchez-Loyo, Correa-Márquez y Luna-Flores (2015), a través de una investigación cualitativa, en el que participaron siete hombres gay, se evidenció la existencia de pensamientos suicidas entre las víctimas.

Cáceres et al. (2011) en otro estudio realizado con 112 estudiantes de la Ciudad de México observó que el 88.39\% manifestaba haber escuchado insultos homofóbicos, el 58.42\% utilizó expresiones homofóbicas en la escuela y el $50.89 \%$ se identificaba como víctima de bullying con expresiones homofóbicas (entre los cuales se encontraban heterosexuales). Asimismo, List (2015) evidenció que en una muestra de 148 estudiantes universitarios el 19.59\% indicaba haber presenciado situaciones de bullying homofóbico entre sus compañeros/as. También Otero (2016) en una muestra de 150 estudiantes universitarios de Nuevo León observó que el $98.67 \%$ reconocía haber presenciado bullying homofóbico en su entorno escolar (especialmente hacia gays), el 33.33\% se identificaba como agresor/a (en mayor medida hacia gays), existía situaciones de bullying homofóbico entre integrantes LGTBI y la mitad de las personas que se identificaban como LGTBI señalaban haber sufrido bullying verbal, psicológico y aislamiento.

Finalmente, también cabe destacar que existen diversos estudios que analizan el calado de las actitudes homófobas, tanto en el estudiantado preuniversitario como en el universitario (Lozano, 2009; Moral de la Rubia \& Martínez-Sulvarán, 2010; 2011; Moral de la Rubia \& Valle de la O, 2011; Delgado \& Young, 2012).

\section{El presente estudio}

Como se ha podido comprobar en el anterior epígrafe, la mayor parte de los estudios realizados en México sobre el bullying homofóbico están contextualizados en la Ciudad de México. También se observa que existe una mayor tendencia a analizar las actitudes LGTBI-fóbicas en el estudiantado que propiamente reconoce la existencia de este tipo de bullying y ser víctima, invisibilizando, así, a quienes no son LGTBI 
y sufren dichos actos o actitudes, los agresores y los espectadores. Es por ello que se plantea una investigación con el objetivo de identificar la existencia y prevalencia del bullying homofóbico en las secundarias del estado de Nuevo León. Asimismo, se proponen como objetivos específicos: (i) analizar la proporción que existe de bullying homofóbico respecto al total en las secundaria, (ii) describir el perfil de las víctimas y de los agresores de este tipo de violencia, (iii) identificar las formas a través de las cuales se materializa la violencia entre iguales, (iv) describir los participantes que se identifican como víctimas del bullying homofóbico, (v) analizar las vías a través de las cuales señalan las víctimas que se produjo la violencia y el perfil de los agresores, (vi) observar cómo reaccionan las víctimas ante el hostigamiento y (vii) describir las consecuencias que tiene el bullying homofóbico en las víctimas.

\section{Método}

Este estudio se realizó según la metodología mixta (cualitativa y cuantitativa) (Sampieri, Collado \& Baptista, 2014) partiendo del paradigma crítico, en la que utilizando un muestreo intencionado se identificó la prevalencia del bullying y el bullying homofóbico en secundarias regiomontanas. Para ello se han seleccionado cuatro centros educativos del Área Metropolitana de Monterrey.

\section{Participantes}

La muestra que formó parte de la investigación estuvo formada por un total de 330 estudiantes del último grado de Secundaria. De los cuales 165 eran hombres (50\%) y 165 mujeres (50\%) con edades comprendidas entre los 15 y los 16 ańos. Participaron alumnos de cuatro escuelas del Área Metropolitana de Monterrey.

Como criterios de inclusión se consideraron los cuestionarios aplicados contestados voluntariamente al completo, por los estudiantes de los centros anteriormente seńalados, matriculados en el último curso de secundaria. Asimismo, para el análisis cualitativo se tomaron en 
Bullying homofóbico en México a nivel de secundaria: el contexto de Nuevo León / Rodríguez O.

consideración aquellos casos en los que el alumnado contestó a las cuestiones de tipo abierto aplicando la saturación teórica.

\section{Medición}

Como instrumentos utilizados en la parte cuantitativa se aplicó un cuestionario de creación propia formado por preguntas abiertas; a través de las cuales se buscaba identificar: (i) las características sociodemográficas de la muestra (sexo, edad, curso académico, centro de estudios y municipio), (ii) la existencia de situaciones de bullying detectadas por los participantes, (iii) la existencia de situaciones de bullying homofóbico detectadas por los participantes, (iv) el perfil de las víctimas y agresores del bullying homofóbico, (v) las formas a través de las cuales se materializa el bullying homofóbico (ver Anexo I). Por otro lado, en la parte cualitativa, se han planteado cuestiones de tipo abierto y de creación propia para identificar en la muestra que se autoidentifica como víctima de bullying homofóbico: (a) la orientación sexual, (b) el perfil de los agresores/as, (c) las formas a través de las cuales se materializa el bullying homofóbico, (c) cómo reaccionó la víctima ante la situación, (d) si intervino el centro escolar y la familia y de qué forma y (e) las consecuencias que tuvo este tipo de acoso/violencia en la víctima (ver Anexo I).

Dicho instrumento se aplicó previamente a una muestra de 50 alumnos de una escuela de Monterrey con objeto de evitar las posibles fuentes de error descritas por García, Gónzález y Maldonado (1999); realizando así los ajustes pertinentes al mismo.

\section{Procedimiento}

El contacto con los participantes se realizó a través de la dirección de los centros, previa solicitud escrita en la que se explicó el motivo, procedimiento y uso de datos de forma anónima en la investigación. Tras la aceptación por parte de los equipos de dirección se acudió a las secundarias entre marzo y abril de 2016, se seleccionaron los grupos del $3^{\circ}$ grado, se presentó la investigación e informó sobre el anonimato 
del instrumento a los alumnos. A continuación, se solicitó su participación y se aplicó de forma voluntaria tanto el consentimiento informado como el instrumento.

\section{Análisis de datos}

Para el análisis de los datos se realizó en la parte cuantitativa una comparación de frecuencias y totales, donde obteniendo los porcentajes de cada cuestión en cada centro educativo se identificaron las variables en las que las frecuencias y totales eran superiores. Asimismo en la parte cualitativa la técnica utilizada fue la de categorización emergente y exposición de cada categoría. De esta forma, se propone alcanzar una triangulación de los datos (Sampieri, Collado \& Baptista, 2014).

Los resultados de la parte cuantitativa se codificaron a través de un programa informático (SPSS) y se expresaron en totales y porcentajes. Asimismo, las cuestiones cualitativas fueron transcritas de forma literal y se procedió a la metodología taxonómica expuesta por Colás (1998): reducción, disposición y transformación de los datos (codificación- definición del sistema de categorías), análisis e interpretación de los mismos, obtención y verificación de conclusiones. En la codificación en cada una de las categorías se han indicado: el centro educativo (S-no de la secundaria), el sexo y número de participante (H-hombres y M-mujeres A, seguido del $\mathrm{n}^{\circ}$ ), la orientación sexual y la edad (G-gay, L-lesbiana, B-bisexual y H-heterosexual, seguido de la edad en $\mathrm{n}^{\circ}$ ), el municipio (Mty-Monterrey, Gua-Guadalupe y SN-San Nicolás) y línea o líneas de la cita (1.). Para garantizar la calidad de la información obtenida se ha utilizado los criterios de confirmabilidad y neutralidad descritos por Noreña, Alcázar-Moreno, Rojas \& Rebolledo-Malpica (2012): transcripciones textuales de las entrevistas y contrastación de los resultados con la literatura existente. 
Bullying homofóbico en México a nivel de secundaria: el contexto de Nuevo León / Rodríguez O.

\section{Resultados y discusión}

\section{Casos de bullying identificados por la muestra}

Ante la cuestión planteada a la muestra en la que se preguntaba si conocían la existencia de algún caso de bullying en la secundaria en la que estudiaban en los últimos 12 meses, tal y como se expresa en la figura 1, se observa que, en la Secundaria A, 45 participantes $(60.81 \%)$ y, en la B, 22 participantes señalan que sí (23.26\%), mientras que en la Secundaria C 37 participantes $(51.38 \%)$ y en la D 43 participantes señalan que sí (40.95\%).

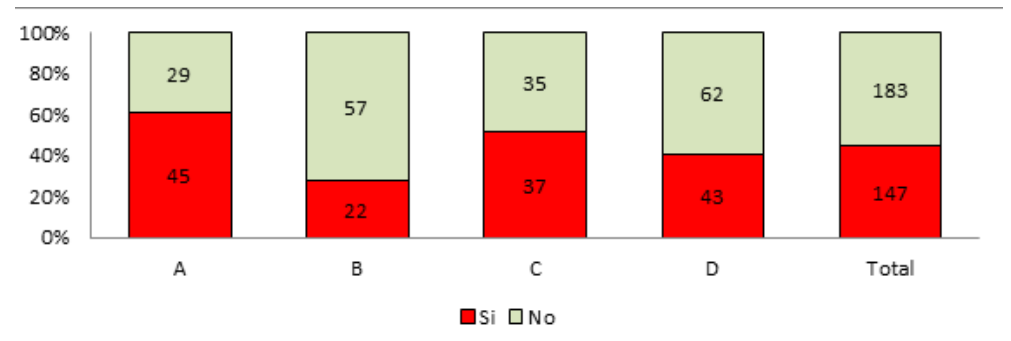

Figura 1. Casos de bullying identificados por la muestra según total y centro educativo.

Estos resultados revelan que tomando en consideración a los 330 participantes, 147 manifiestan que han presenciado alguna situación de bullying, lo cual representa el $44.54 \%$ de la muestra. Se observa que este resultado arroja un elevado nivel de violencia en las escuelas. Este es muy superior al que se identifica en investigaciones precedentes a nivel internacional, en las que se estima que el bullying afecta a más de un 20\% del estudiantado de primaria y secundaria (Carozo, Zapata \& Benites, 2012; De la Vega \& Vallejo, 2012; Del Tronco \& Madrigal, 2013), así como las realizadas en México, en las que los porcentajes oscilan dependiendo del estado, aunque son en todas inferiores al $30 \%$ (Albores-Gallo, Sauceda-García \& Ruiz-Vela, 2011; Loredo-Abdalá, Perea-Martínez \& López, 2008; Joffre-Velázquez et al., 2011; Vega, González, Valle, Flores \& Vega, 2013). 


\section{Casos de bullying homofóbico}

Respecto a la existencia de casos de bullying homofóbico en los últimos 12 meses de las secundarias analizadas, como se observa en el Figura $\mathrm{n}^{\circ} 2$, un total de 46 participantes afirman haberlo presenciado. De estos, 16 son de la secundaria A, 7 tanto de la secundaria B como de la secundaria C y 16 de la secundaria D.

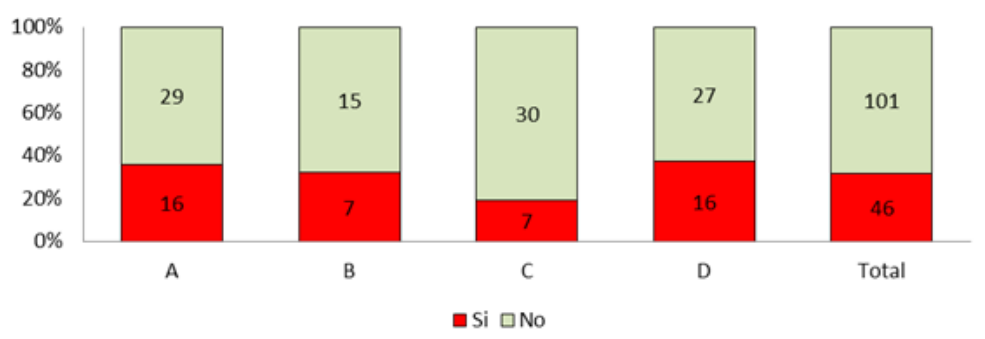

Figura 2. Casos de Bullying homofóbico respecto a los casos totales de bullying identificados por la muestra según total y centro educativo.

Tomando en consideración al alumnado de manifestaba haber presenciado bullying en su centro en los últimos 12 meses se observa que el bullying homofóbico representa el 31,29\% de los distintos tipos de bullying, siendo esta proporción del 35,55\% en la Secundaria A, el $31,81 \%$ en la B, el $18,91 \%$ en la C y el $37,21 \%$ en la D. Asimismo, tomando en consideración la totalidad de participantes es este estudio (330 alumnos) se observa que el 13,93\% manifiesta la existencia de bullying homofóbico en su centro escolar.

Realizando una comparación con estudios precedentes se observa que este resultado es inferior a los evidenciados en estudios tanto a nivel internacional (Gualdi et al., 2008; Martín-Martín, MolinuevoPuras, Pichardo-Galán, Rodríguez-Medina \& Romero-López, 2007; COGAM, 2013; Gehitu, 2012; Prati, D’Augelli \& Pietrantoni, 2011) como en el contexto mexicano (México, 2009; Quintanilla, SánchezLoyo, Correa-Márquez \& Luna-Flores, 2015; Cáceres et al., 2011; Otero, 2016). 
En relación con el perfil de las víctimas del bullying homofóbico, se observa en la tabla 1, la muestra señala que son los gays (50\%), lesbianas $(24 \%)$ o ambos $(18 \%)$ —especialmente gays - quienes reciben principalmente este tipo de acoso, aunque también se evidencia tanto en bisexuales hombres y mujeres (4\%) como en heterosexuales hombres $(4 \%)$.

\section{Tabla 1}

Perfil de víctima y agresor de los casos de bullying homofóbico identificados por la muestra según centro educativo

\begin{tabular}{lccccc}
\hline & \multicolumn{5}{l}{ Secundaria } \\
\cline { 2 - 6 } & $\mathrm{N}$ & $\mathrm{A}$ & $\mathrm{B}$ & $\mathrm{C}$ & $\mathrm{D}$ \\
\hline Víctima & 23 & 9 & 3 & 6 & 5 \\
$\quad$ Gay & 11 & 2 & 3 & 1 & 5 \\
Lesbiana & 8 & 3 & 1 & 0 & 4 \\
Gays y lesbianas & & & & & \\
Bisexual & 1 & 0 & 0 & 0 & 1 \\
$\quad$ Hombres & 1 & 1 & 0 & 0 & 0 \\
$\quad$ Mujeres & & & & & \\
Homosexual & 2 & 1 & 0 & 0 & 1 \\
$\quad$ Hombres & 0 & 0 & 0 & 0 & 0 \\
$\quad$ Mujeres & 46 & 16 & 7 & 7 & 16 \\
TOTAL & & & & & \\
Agresor & 19 & 8 & 3 & 5 & 3 \\
Hombres & 1 & 1 & 0 & 0 & 0 \\
Mujeres & 2 & 0 & 1 & 0 & 1 \\
Hombres y mujeres & 24 & 7 & 3 & 2 & 12 \\
No especifica & 46 & 16 & 7 & 7 & 16 \\
TOTAL & & & & &
\end{tabular}


Respecto al perfil de los agresores se observa que 24 participantes $(52 \%)$ no especifica si se trata de hombres, mujeres o ambos (ver tabla 1). Considerando a quienes si especifican el sexo del agresor se observa que son los hombres (86\%) quienes ejercen este tipo de bullying principalmente, aunque también se corrobora su existencia ejercida a través de mujeres (5\%) e indistintamente por ambos sexos (9\%).

Se identifica que los hombres son el principal perfil tanto de víctimas como de agresores de este tipo de bullying, aunque este aspecto también se ponen de manifiesto en investigaciones precedentes tales como las realizadas por Gualdi et al. (2008), FELGTB y COGAM (2013), Martín-Martín, Molinuevo-Puras, Pichardo-Galán, RodríguezMedina y Romero-López (2007), Generelo, Garchitorena, Montero e Hidalgo (2012), Martxueta y Etxeberria (2014), López Amurri (2013) y Gehitu (2012) a nivel internacional, así como otras contextualizadas en México (Lozano, 2009; 2014; Otero, 2016; Granados-Cosme \& Delgado-Sánchez, 2008; Ortiz \& Granados, 2003; Cáceres et al., 2011).

Finalmente, respecto a las formas a través de las cuales se ejerce este tipo de violencia se observa que es mediante insultos (27 participantes), burlas (5), golpes (8), aislamiento (2 participantes), ciberllullying (4), críticas (2), molestar (1), rumores (1) y hacer sentir mal (1). Asimismo, se observa que 8 participantes no contestan a esta cuestión (ver Figura 3).

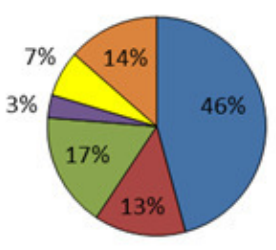

$\square$ Violencia verbal
$\square$ Violencia física
$\square$ Violecnia psicológica
$\square$ Aislamiento
$\square$ Ciberbullying
$\square$ No especifica

Figura 3. Vías de ejecución del bullying homofóbico identificados por la muestra en $\%$. 
Estos resultados evidencian que la violencia verbal es la que representa la principal vía a través de la cual se materializa el bullying homofóbico. No obstante, también se pone de manifiesto la existencia de violencia psicológica y física, así como del aislamiento (aunque en menor medida) y el uso de las nuevas tecnologías (ciberbullying). Cabe señalar que estos resultados corroboran los expuestos anteriormente a través de las investigaciones de Granados-Cosme y Delgado-Núñez (2008), Lozano y Rocha (2011) y Otero (2016) en el contexto mexicano y las de Gualdi et al. (2008), Martxueta y Etxeberría (2014), COGAM (2013) o FELGTBI (2007) a nivel internacional.

\section{Victimas de bullying homofóbico}

Se observa que de los 330 participantes, 7 estudiantes $(2,12 \%)$ se identifican como víctimas de bullying homofóbico, de los cuales 2 son hombres y 5 mujeres. Respecto a los centros educativos analizados: 2 son de la Secundaria A, 3 de C y 2 de la D. Asimismo, se identifica en la de la Secundaria no 99 de Guadalupe ningún alumno/a seńala haber sufrido este tipo de acoso/violencia (ver tabla 2).

En referencia a la orientación sexual de las víctimas se observa que 5 se identifican como lesbianas, 1 como gay y otro como hombre heterosexual. Del mismo modo, 3 indican que poseen 14 años y 5 de ellos 15 ańos. Respecto a las formas a través de las cuales se produjo el bullying homofóbico 3 participantes señalan que fue mediante ciberbullying, 3 a través de insultos (violencia verbal), 1 mediante rumores y acoso (violencia psicológica), otro por golpes (violencia física) y otro más mediante aislamiento. Igualmente, se observa que en 3 participantes se pone de manifiesto la existencia de la combinación de distintas formas de hostigamiento (ver tabla 2). 


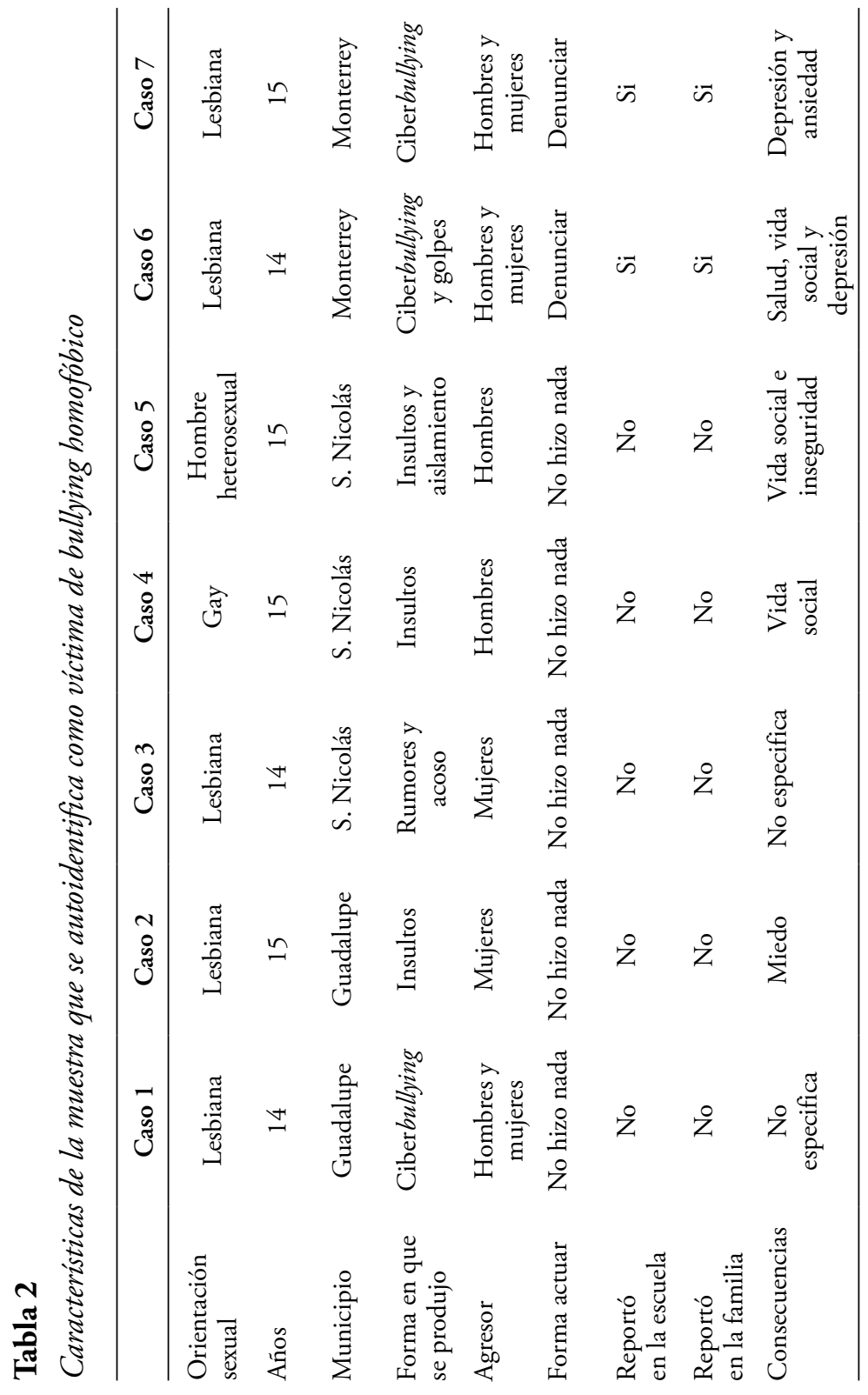


Bullying homofóbico en México a nivel de secundaria: el contexto de Nuevo León / Rodríguez O.

Así, se identifican manifestaciones del tipo:

"Subieron fotos mías a internet y las modificaron. Aparecía besándome con una chica y tenía letras que decian lesbiana, lesbiana, lesbiana. Todos se reían de mí y ponian comentarios" (S25, M38, L14, Gua, 1.18-19). Caso 1.

"Me insultan y me llaman lesbiana, enferma y cosas asi" (S25, M61, L15, Gua, 1.21). Caso 2.

"Les dicen a mis amigas que no se acerquen porque les quiero hacer algo y que como me gustan las mujeres pues les dicen que estoy enamorada de ellas para que no se acerquen. También me persiguen al salir de clases $y$ me retan" (S46, M12, L14, SN, 1.19-21). Caso 3.

"Me llaman joto, gay, niña..."(S46, H22, G15, SN, 1.21). Caso 4.

"Dicen que soy gay aunque no lo soy porque en la escuela juego con niñas y cuando me acerco a algún chavo de la clase no me hablan y no quieren acercarse a mi porque dicen que soy gay" (S46, H46, H15, SN, 1.22-24). Caso 5.

"Me insultan por el facebook diciéndome lesbiana, puta y cosas asi y en la escuela me tiran golpes" (S30, M8, L14, Mty, 1.20-21). Caso 6.

"Hicieron una página en la que ponen fotos mías y me insultan ahí diciendo que soy lesbiana y que nadie se acerque a mi" (S30, M29, L15, Mty, 1.22-23). Caso 7.

Respecto a los agresores, dos participantes señalan que son hombres, otros dos que son mujeres y tres que son tanto hombres como mujeres. Por otro lado, se observa que solamente dos participantes (ambas mujeres lesbianas de la Secundaria no 30 de Monterrey) manifiestan que denunciaron los hechos tanto en su familia como en la escuela, mientras que las restantes cinco víctimas indican que no realizaron nada al respecto.

"Se lo dije a mis padres y fueron a la escuela a hablar con mis profesores (...) Los maestros les llamaron la atención a mis compañeros" (S30, M8, L14, Mty, 1.23-26). Caso 6. 
"Hablé con una profesora y se lo conté a mi tía (...) La directora llamó a mi tía y platicaron y después obligaron a mis compañeros a cerrar la página"(S30, M29, L15, Mty, 1.25-28). Caso 7.

Realizando un análisis respecto al sexo de las víctimas y las tipologías de violencia se destaca que hacia los hombres se produce violencia verbal y psicológica, mientras que en las mujeres se identifica tanto violencia verbal, psicológica y física como ciberbullying. Asimismo, se observa que hacia los hombres se manifiesta que los agresores son hombres y hacia las mujeres compañeras y/o estudiantes de ambos sexos. Por otro lado, se aprecia que existe un bajo nivel de denuncias, siendo mayor en mujeres.

Finalmente, se observa que en cinco estudiantes se evidencia la existencia de consecuencias derivadas del hostigamiento y/o violencia a la que fueron sometidos/as, la que hace referencia a cuestiones psicológicas (inseguridad, miedo, ansiedad y depresión), al estado de salud y/o al aislamiento social (vida social). Así, se identifican manifestaciones tales como:

"Me daba miedo ir a la escuela y no quería ir" (S25, M61, L15, Gua, 1.23). Caso 2.

"Me afectó en mi vida social porque no sabia cómo hablarles a los chavos de clase por si me pegaban" (S46, H22, G15, SN, 1.24-25). Caso 4.

"Soy muy inseguro y no tengo amigos hombres en la escuela porque no quieren que los vean conmigo" (S46, H46, H15, SN, 1.27-28). Caso 5.

"En mi salud, en mi vida social y siempre estaba deprimida y no quería ir a la escuela" (S30, M8, L14, Mty, 1.28). Caso 6.

"Cuando pasó eso tenía miedo de contarlo en la escuela, estaba muy nerviosa como me encontraba mal como angustiada y no quería ir a la escuela. Siempre estaba triste y no tenía ganas de hacer nada" (S30, M29, L15, Mty, 1.31-33). Caso 7. 
Bullying homofóbico en México a nivel de secundaria: el contexto de Nuevo León / Rodríguez O.

Se observa, por tanto, que el bullying homofóbico, tal y como señalan autores en investigaciones precedentes, afecta tanto a personas LGTBI como a heterosexuales (Gualdi et al., 2008; Samaniego \& Bermúdez, 2015; Agustín, 2009), aunque, como se ha señalado anteriormente, solamente se identifican víctimas gays, lesbianas y heterosexuales a través del análisis cualitativo. Respecto a los agresores, al igual que señalan autores como Gualdi et al. (2008), Otero (2016), Martxueta y Etxeberría (2014) y De Souza, Pereira y Ferro (2015) se ejerce tanto por hombres como por mujeres. Sin embargo, estos estudios señalan que son los hombres quienes reportan un mayor nivel de violencia. Mientras que a través del análisis cualitativo la muestra señala que se produce mayormente por mujeres.

Respecto a las formas de violencia manifestadas se ha constatado que el bullying homofóbico puede tomar diversas caras, siendo la más común la violencia verbal, aspecto que también se identifica en los estudios de Gualdi et al. (2008), Otero (2016), Martxueta y Etxeberría (2014), Granados-Cosme y Delgado-Sánchez (2008), Cáceres et al. (2011), COGAM (2013) y De Souza, Pereira y Ferro (2015). Por otro lado, cabe señalar que como se evidencia en otras investigaciones existe un bajo nivel de denuncias, resultando clave en estos casos la actuación tanto del centro como del entorno familiar (Gualdi et al., 2008; FELGTBI, 2007; COGAM, 2013; Agustín, 2009).

Finalmente, también se constata mediante este análisis cualitativo que, como indican diversos autores, este tipo de hostigamiento/ violencia se caracteriza por derivar en distintas consecuencias en las víctimas tales como miedo, inseguridad, depresión, ansiedad, deterioro de la salud física y afecciones en la vida social-relacional (Burton, Marshal \& Chisolm, 2014; Pachankis, Goldfried \& Ramrathan, 2008; Martxueta \& Etxeberría, 2014; Antonio, Pinto, Pereira, Farcos \& Moleiro, 2012). No obstante no se identifican otros, tales como el empeoramiento del rendimiento académicos, el fracaso y la deserción escolar (Poteat et al., 2011; Burton, Marshal \& Chisolm, 2014) y mayor riesgo de suicidio o intento de llevar a cabo conductas asociadas (Poteat et al. 2011; Baiocco et al., 2015). 


\section{Limitaciones}

Tomando en consideración que, según indica la Secretaría de Educación Pública de México (SEP, 2016), en el curso académico 2015-2016, el número de alumnos de Secundaria en el Estado de Nuevo León fue de 289.077 alumnos (universo). Siendo la muestra de la presente investigación de 330 estudiantes y tomando un intervalo de confianza del $95 \%$, el margen de error resultante corresponde a un $5,4 \%$. Es por ello que los resultados no son representativos, pero si suficientes para analizar el estado del problema de forma exploratoria, siendo el contexto regiomontano en enclave en el que no se realizaron investigaciones precedentes al respecto. Asimismo, al optar por una metodología mixta, los datos cualitativos permiten realizar una triangulación de los resultados.

Por otro lado, también es necesario destacar que al tratarse en la parte cualitativa de un muestreo de voluntarios, pudieran existir personas que, siendo víctimas de bullying LGTBI-fóbico, no se identifiquen o quieran identificarse como tal, especialmente las personas heterosexuales que lo sufren y que no asumen su orientación sexual o identidad de género y quienes no quieran declararlo.

\section{Conclusión}

A través de esta investigación se ha podido constatar que el bullying homofóbico es una realidad palpable en las secundarias regiomontanas. Asimismo, se identifica que el entorno escolar y el sistema familiar son elementos claves para prevenir este tipo de violencia y posibles consecuencias en las víctimas. No obstante, sería conveniente realizar un estudio representativo que incluyese alumnado de todos los municipios del estado y no solo del Área Metropolitana de Monterrey (incluyendo así población rural), así como analizar las variables de sexo, orientación e identidad sexual de la muestra. Por otra parte, incluir una segunda parte cualitativa a través de historias de vida de víctimas, pudiera ser un elemento añadido en la triangulación de resultados. 
La existencia de políticas educativas destinadas a prevenir la existencia de este tipo de bullying que no solo afecta a personas LGBTI se considera un elemento esencial en la lucha contra la LGTBI-fobia que pude tener cabida en el espacio escolar. Es por ello que se considera necesario promover una ley integral de lucha contra este tipo de discriminación y el desarrollo de planes y programas específicos que tengan como objeto prevenir el bullying homofóbico, concienciar a la población en general y específicamente a las familias y el entorno educativo, así como dotar a los centros educativos de profesionales de referencia —entre los que deberían de estar trabajadores/as socialesque brinden el apoyo necesario a las víctimas.

Finalmente cabe señalar que, puesto que la FITS en el año 2014 aprobó la creación de un banco internacional de buenas prácticas en materia de identidad sexual y trabajo social (CGTS, 2014), es necesario promover dentro de las profesiones en el campo de la salud y del trabajo social un espacio de debate y reflexión respecto al papel que los trabajadores/as sociales deben tomar ante esta problemática identificada por la Organización Mundial de la Salud como un problema de salud pública.

\section{Referencias}

Agustín, S. (2009). Diversidad sexual en las aulas. Evitar el bullying o acoso homofóbico. Badajoz: Fundación Triángulo y Plural.

Albores-Gallo, L., Sauceda-García, J. M. \& Ruiz-Vela, S. (2011). El acoso escolar (bullying) y su asociación con trastornos psiquiátricos en una muestra de escolares en México. Salud Pública de México, 53(3), 220-227.

Antonio, R., Pinto, T., Pereira, C., Farcos, D. \& Moleiro, C. (2012). Bullying homofóbico no contexto escolar en Portugal. Psicología, 26(1), 17-32. https://doi.org/10.17575/rpsicol.v26i1.260 
Bastida, L. (2010). Homofobia en escuelas, una realidad cercana. La Jornada. UNAM. Recuperado el 8 de junio de 2015 de http:// www.jornada.unam.mx/2010/05/06/lsportada.html

Bastida, L. (2012). La violencia hacia lo diferente. El acoso escolar por homofobia: una realidad en las escuelas mexicanas. Rayuela. Revista Iberoamericana sobre Niñez y Juventud en Lucha, 3(6), 102-109.

Baiocco, R., Ioverno, S., Lonigro, A., Baumgartner, E. \& Laghi, F. (2015). Suicidal Ideation among Italian and Spanish Young Adults: The Role of Sexual Orientation. Archives of suicide research, 19(1), 75-88. https://doi.org/10.1080/13811118.201 3.833150

Birkett, M., Espelage, D., L. \& Koenig, B. (2009). LGB and questioning students in schools: the moderating effects of homophobic bullying and school climate on negative outcomes. Journal Youth Adolesc, 38(7), 989-1000. https://doi.org/10.1007/ s10964-008-9389-1

Burton, C. M., Marshal, M. P. \& Chisolm, D. J. (2014). School absenteeism and mental health among sexual minority youth and heterosexual youth. Journal of School Psychology, 52(1), 37-47. https://doi.org/10.1016/j.jsp.2013.12.001

Cáceres, C. F., Silva-Santisteban, A., Salazar, X., Cuadros, J., Olivos, F. \& Segura, E. (2011). Estudio a través de Internet sobre "Bullying" y sus manifestaciones homofóbicas en escuelas de Chile, Guatemala, México y Perú, y su impacto en la salud de jóvenes varones entre 18 y 24 años. Informe Final. Lima: Universidad Peruana Cayetano Heredia.

Carozo, J. C., Zapata, L. \& Benites, L. (2012). ¿Es la escuela un lugar seguro? En M. E. Murueta y M. Orozco, Psicología de la violencia. Tomo II (pp. 143-166). México D. F. : AMAPSI.

CGTS (2014). La FITS aprueba la propuesta sobre "Homosexualidady TrabajoSocial"delCGTS. Recuperadoel 18demayode2016enhttps:// www.cgtrabajosocial.es/consejo/noticias/la-fits-aprueba-la- 
Bullying homofóbico en México a nivel de secundaria: el contexto de Nuevo León / Rodríguez O.

propuesta-sobre-homosexualidad-y-trabajo-social-del-cgts/ 2232/view

Cobo, P. \& Tello, R. (2010). Bullying. El acecho cotidiano en las escuelas. México D.F.: Limusa.

COGAM (2013). Homofobia en las aulas: ¿Educamos en la diversidad afectivo sexual? Madrid: FELGTBI. Recuperado el 4 de mayo de 2016 de http://www.felgtb.org/temas/educacion/ documentacion/investigaciones/i/5433/449/cogam-homofobiaen-las-aulas-2013

Colás, P. (1998). El análisis cualitativo de datos. En L. Buendía, P. Colás y F. Hernández, Métodos de investigación en Psicopedagogía (pp. 288-313). Madrid: McGraw-Hill Interamericana de España.

D’Augelli, A. R., Pilkington, N. W. \& Hershberger, S. L. (2002). Incidence and Mental Health Impact of Sexual Orientation Victimization of Lesbian, Gay, and Bisexual Youths in High School. School Psychology Quarterly, 17(2), 148-167. https://doi. org/10.1521/scpq.17.2.148.20854

Delgado, S. \& Young, C. (2012). Diferencias de actitud ante la homosexualidad entre los estudiantes universitarios de la Ciudad de México. Odiseo Revista electrónica de pedagogía, 9, 18.

De la Vega, R. \& Vallejo, R. (2012). La noción de la violencia: un concepto complejo de construir en la etapa adolescente. En M. E. Murueta y M. Orozco, Psicología de la violencia. Tomo II (pp. 113-128). México D. F.: AMAPSI.

Del Tronco, J. \& Madrigal, A. (2013). Violencia escolar en México: una exploración de sus dimensiones y consecuencias. Revista Trabajo Social UNAM, 7(4), 9-27.

De Souza, J. M. D., Pereira, J. P. D. \& Faro, A. (2015). Bullying and Homophobia: Theoretical and Empirical Approaches. Psicologia Escolar e Educacional, 19(2), 289-298. https://doi. org/10.1590/2175-3539/2015/0192837

FELGTBI (2007). El absentismo escolar por causa de la orientación sexual o la identidad de género. Recuperado el 8 de mayo de 2016 
en http://www.cogam.es/secciones/educacion/i/57703/153/ absentismo-escolar-y-orientacion-sexual-e-identidad-de-genero FELGTB \& COGAM (2013). Acoso escolar (y riesgo de suicidio) por orientación sexual e identidad de género: Fracaso del Sistema Educativo. Madrid, FELGTB. Recuperado el 8 de mayo de 2016 en http://www.felgtb.org/temas/educacion/documentacion/ investigaciones/i/3363/449/acoso-escolar-y-riesgo-de-suicidiopor-orientacion-sexual-e-identidad-de-genero-fracaso-del-sistema-educativo

García, M. (2015). Orientación sexual y homofobia en adolescentes españoles. (Trabajo Fin de Grado en Psicología). Barcelona: Universitat Jaume I.

García, P.A.; Gónzález, A. \& Maldonado, J. A. (1999). Problemas en el Diseño y Validación de Cuestionarios: tratamiento con QUESTPOT v.1.2. Estadistica Española, 41(144), 19-46.

Gehitu (2012). Opiniones sobre la diversidad afectivo-sexual (Gehitu 2011-2012). Estudio sobre la diversidad afectivo-sexual entre el alumnado de $3^{\circ}$ y $4^{o}$ de ESO en la provincia de Gipuzkoa. Recuperado el 11 de mayo de 2016 de http://www.felgtb.org/ temas/educacion/documentacion/investigaciones/i/3532/449/ opiniones-sobre-la-diversidad-afectivo-sexual-gehitu-2011-2012 Generelo, J., Garchitorena, M., Montero, P. \& Hidalgo, P. (2012). Acoso escolar homofóbico y riesgo de suicidio en adolescentes y jóvenes $L G B$. Madrid: COGAM.

Granados-Cosme, J. A. \& Delgado-Sánchez, G. (2008). Identidad y riesgos para la salud mental de jóvenes gays en México: recreando la experiencia homosexual. Cuaderno Saúde Pública, 24(5), 10421050. https://doi.org/10.1590/S0102-311X2008000500011

Gualdi, M., Martelli, M., Wilhelm, W., Biedroń R., Graglia, M. \& Pietrantoni, L. (2008). Bullismo nelle scuole: manuale insegnanti. Bologna: Arcigay. Recuperado en http://www.arcigay.it/wp-content/uploads/2008-Schoolmate-Manuale-per-insegnanti1.pdf Joffre-Velázquez, V. M., García-Maldonado, G., Saldívar-González, A. H., Martínez-Perales, G., Lin-Ochoa, D. \& Quintanar- 
Bullying homofóbico en México a nivel de secundaria: el contexto de Nuevo León / Rodríguez O.

Martínez, S. (2011). Bullying en alumnos de secundaria. Características generales y factores asociados al riesgo. Boletín médico del Hospital Infantil de México, 68(3), 193-202.

Kolbert, J. B., Crothers, L. M., Bundick, M. J., Wells, D. S., Buzgon, J., Berbary, C. \& Senko, K. (2015). Teachers' Perceptions of Bullying of Lesbian, Gay, Bisexual, Transgender, and Questioning (LGBTQ) Students in a Southwestern Pennsylvania Sample. Behavioral Sciences, 5(2), 247-263.

List, M. (2015). Los universitarios frente a la homofobia. El caso de la Benemérita Universidad Autónoma de Puebla. Sinética. Revista electrónica de educación, 46, 1-15.

Loredo-Abdalá, A., Perea-Martínez, A. \& López, G. E. (2008). "Bullying": acoso escolar. La violencia entre iguales. Problemática real. Acta Pediátrica de México, 29(4), 210-214.

Lozano, I. (2009). El significado de la homosexualidad en jóvenes de la ciudad de México. Enseñanza e Investigación en Psicología, 14(1), 153-168.

Lozano, I. (2014). Violencia institucional homofóbica y emociones de hombres gay de la Ciudad de México. Revista Puertorriqueña de Psicología, 25(2), 298-312.

Lozano, I. \& Rocha, T. E. (2011). La homofobia y su relación con la masculinidad hegemónica en México. Revista Puertorriqueña de Psicología, 22(2), 101-121.

López-Amurri, E. (Coord.) (2013). Homofobia en las aulas: ¿educamos en la diversidad afectivo sexual? Madrid: COGAM. Recuperado el 9 de mayo de 2016 de http://www.cogam.es/ secciones/educacion/documentos-sin-orden/i/1126824/154/ homofobia-en-las-aulas-2013

Martín Martín, N., Molinuevo Puras, B., Pichardo Galán, J. I., Rodríguez Medina, P. O. \& Romero López, M. (2007). Actitudes ante la diversidad sexual de la población adolescente de Coslada (Madrid) y San Bartolomé de Tirajana (Gran Canaria). Madrid, FELGTB. Recuperado el 10 de mayo de 2016 de http://www.cogam.es/secciones/educacion/i/144087/153/ 
investigacion-actitudes-ante-la-diversidad-sexual-de-lapoblacion-adolescente-de-coslada-y-san-bartolome-de-tirajana Martxueta, A. \& Etxeberria, J. (2014). Análisis diferencial retrospectivo de las variables de salud mental en lesbianas, gais y bisexuales (LGB) víctimas de bullying homofóbico en la escuela. Revista de Psicopatología y Psicología Clinica, 19(1), 23-35. https://doi. org/10.5944/rppc.vol.19.num.1.2014.12980

México (2009). Informe Nacional sobre Violencia de Género en la educación básica en México. México: Secretaría de Educación Pública y Unicef.

Moral de la Rubia, J. \& Martínez, J. O. (2010). Validación de una Escala de Homofobia creada en México. Revista Latinoamericana de Medicina Conductual, 1(1), 99-108. https://doi.org/10.5461/ rlmc.v1.n1.11

Moral de la Rubia, J. \& Valle de la O, A. (2011). Validación de la Escala Mexicana de Homofobia EHF-6. Psicología Iberoamericana, 19(1), 80-88.

Moratto, N. S., Cárdenas, N. \& Berbesí, D. Y. (2012). Validación de un cuestionario breve para detectar intimidación escolar. Revista CES Psicología, 5(2), 70-78.

Mooij, T. (2015). Relevance of student and contextual school variables in explaining a student's severity of violence experienced. Teachers and Teaching, 21(8), 926-940. https://doi.org/10.1080 /13540602.2015.1005864

Noreña, A. L., Alcázar-Moreno, N., Rojas, J. \& Rebolledo-Malpica, D. (2012). Aplicabilidad de los criterios de rigor y éticos en la investigación cualitativa. Aquichán, 13(3), 263-274.

Olweus, D. (1998). Acoso escolar, bullying, en las escuelas: hechos e intervenciones. Bergen: Universidad de Bergen.

Ortiz, L. \& Granados, J. (2003). Violencia hacia bisexuales, lesbianas y homosexuales de la Ciudad de México. Revista Mexicana de Sociología, 65(2), 265-303. Recuperado el 12 de mayo de 2016 de http://www.ejournal.unam.mx/rms/2003-2/RMS03201.pdf? pagewanted $=$ all 
Bullying homofóbico en México a nivel de secundaria: el contexto de Nuevo León / Rodríguez O.

Otero, L. M. (2016). Bullying homofóbico en el contexto mexicano: voces desde estudiantes de trabajo social regiomontanos. Trabajo Social Hoy, 77, 41-62. https://doi.org/10.12960/TSH. 2016.0003

Pachankis, J.E., Goldfried, M. R. \& Ramrattan, M.E. (2008). Extension of the rejection sensitivity construct to the interpersonal functioning of gay men. Journal of Consulting and Clinical Psychology, 76(2), 306. https://doi.org/10.1037/0022-006X.76.2.306

Poteat, V. P. \& Espelage, D. L. (2005). Exploring the relation between bullying and homophobic verbal content: The Homophobic Content Agent Target (HCAT) Scale. Violence and Victims, 20, 513-528. https://doi.org/10.1891/0886-6708.2005.20.5.513

Poteat, V. P., Mereish, E. H., Digiovanni, C. D. \& Koenig, B. W. (2011). The effects of general and homophobic victimization on adolescents' psychosocial and educational concerns: the importance of intersecting identities and parent support. Journal of Counseling Psychology, 58, 597-609. https://doi.org/10.1037/ a0025095

Prati, G., D'Augelli, A. R. \& Pietrantoni, L. (2011). Aspects of Homophobia in Italian High Schools: Students' Attitudes and Perceptions of School Climate. Journal of Applied Social Psychology, 41(11), 2600-2620. https://doi. org/10.1111/j.1559-1816.2011.00842.x

Quintanilla, R., Sánchez-Loyo, L. M., Correa-Márquez, P. \& LunaFlores, F. (2015). Proceso de aceptación de la homosexualidad y la homofobia asociados a la conducta suicida en varones homosexuales. Masculinities and Social Change, 4(1), 1-25.

Rivers, I. (2001). The bullying of sexual minorities at school: Its nature and long-term correlates. Educational and Child Psychology, $18(1), 32-46$.

Rivers, I. \& Soutter, A. (1996). Bullying and the Steiner School Ethos A Case Study Analysis of a Group-centred Educational Philosophy. School Psychology International, 17(4), 359-377. https:// doi.org/10.1177/0143034396174005 
Samaniego, J. L. \& Bermúdez, D. A. (2015). Discriminación hacia jóvenes homosexuales en su entorno educativo. (Tesis de Licenciatura). Cuenca: Universidad de Cuenca. Recuperado el 15 de febrero de 2016 de http://dspace.ucuenca.edu.ec/ handle/123456789/23311

Sampieri, R., Collado, C. \& Baptista, P. (2014). Metodología de la investigación. México DF: McGrawHill.

Secretaria de Educación Pública (2016). Estadística del Sistema Educativo. Nuevo León. Ciclo escolar 2015-2016. Recuperado el 5 de abril de 2017 de http://www.snie.sep.gob.mx/ descargas/estadistica_e_indicadores/estadistica_e_indicadores_ educativos_19NL.pdf

Serrate, R. (2007). Bullying, acoso escolar. Guía para entender y prevenir el fenómeno de la vioelncia en las aulas. Madrid: Laberinto.

Vega, J. A. (2013). La pandemia del bullying en México: políticas sociales urgentes. Revistas Trabajo Social UNAM, 7(4), 71-87.

Vega, M. G., González, G. J., Valle, M. A., Flores, M. E. \& Vega, A. (2013). Acoso escolar en la zona metropolitana de Guadalajara, México: prevalencia y factores asociados. Salud Colectiva, 9(12), 183-194. https://doi.org/10.18294/sc.2013.31

Recibido: 31 de mayo, 2016

Revisado: 14 de febrero, 2018

Aceptado: 27 de abril, 2018 
Bullying homofóbico en México a nivel de secundaria: el contexto de Nuevo León / Rodríguez O.

\section{Anexo I. Cuestionario aplicado}

A continuación se presenta un cuestionario, el cual nos gustaría que leyeses detenidamente y aceptases participar de forma voluntaria. Este documento es anónimo y tendrá un uso única y exclusivamente de carácter científico. Puesto que los resultados serán publicados respetando las normas de confidencialidad.

\section{DATOS SOCIODEMOGRÁFICOS}

SEXO:

$\square$ Hombre

$\square$ Mujer

Edad:

\section{Municipio:}

Secundaria:

Curso:

\section{VIOLENCIA EN LA ESCUELA}

1. ¿Se ha producido alguna situación de bullying en tu colegio en los últimos 12 meses?

Sí

No

2. ¿Se ha producido bullying en tu colegio hacia alguien que es o se presuponía que era gay, lesbiana o bisexual en los últimos 12 meses?

Sí

No

2.1. ¿Cómo se produjo?

2.2. ¿Cómo era el agresor/a?

Hombre

Mujer

Hombres

Mujeres

Hombres y mujeres

2.3. ¿Cuál era el sexo de la víctima/s?

Hombre/s

Mujer/s

Hombres y mujeres

2.3. ¿Cuál era la orientación sexual de la víctima?

Gay/s

Lesbiana/s

Gays y lesbianas

Bisexual/es

Heterosexual/es
3. ¿Alguna vez has sufrido bullying en tu colegio por ser o presuponer que eras gay, lesbiana o bisexual?

$\square$ Sí

No

3.1. ¿Con cuál de las siguientes opciones te identificas?

Gay

Lesbiana

Bisexual

Heterosexual/es

3.2. ¿Cómo se produjo?

3.3. ¿Qué hiciste?

3.4. ¿Te afectó el bullying? (estado anímico, salud, rendimiento académico, vida social, etc.)

3.5. ¿¿e lo dijiste al centro? ¿Qué hizo al respecto?

3.6. ¿Se lo dijiste a tu familia? ¿Qué hizo al respecto? 